\title{
Eletroanálise do Ponceau 4R utilizando um eletrodo de pasta de carbono modificado com hexacianoferrato de níquel
}

\author{
Francisco Damião de Mendonça* (Graduando em Química na UERN) \\ Janete Jane A. Fernandes ( Prof.Ad. do DQ e do PPGCN na UERN) \\ Thiago Mielle B.F. Oliveira ( TNS e Prof. do quadro do PPGCN na UERN)
}

*E-mail: dammiao_mendonca@hotmail.com

\begin{abstract}
Resumo:
Os agravamentos dos problemas ambientais, em decorrências das atividades indústrias, têm aumentado a demanda por novas tecnologias para o monitoramento dos poluentes gerados. Neste âmbito, incluem-se as indústrias que utilizam corantes como matéria-prima, já que esses compostos podem desequilibrar seriamente os ecossistemas, além de afetar a saúde dos seres vivos, quando descartados de forma inapropriada. Os azo-compostos, como o Ponceau 4R (E124), estão entre os mais utilizados nos setores têxtil, farmacêutico e alimentício, mostrando a importância do desenvolvimento de métodos para a detecção e quantificação desses potenciais contaminantes. Neste trabalho, um eletrodo de pasta de carbono quimicamente modificado com um filme de hexacianoferrato de níquel (Ni-HCF/EPC) foi utilizado para a eletroanálise de E124 em águas naturais. Entre as principais vantagens observadas para este dispositivo, destacam-se a elevada estabilidade, sensibilidade e precisão para a análise do corante. Utilizando a cronoamperometria como técnica eletroanalítica, foi possível quantificar E-124 com um limite de detecção de $9,90 \times 10^{-7} \mathrm{~mol} \mathrm{~L}^{-1}$. As porcentagens de recuperação obtidas em amostras de águas naturais artificialmente contaminadas, contendo diferentes níveis de matéria orgânica, variaram de 85,9 a 104,2 \%, mostrando a aplicabilidade do procedimento proposto.
\end{abstract}

Palavras-chave: Águas naturais; Azo-compostos; Ponceau 4R; Eletrodos quimicamente modificados; Eletroanálise.

\section{Introdução}

De um modo geral, os efluentes industriais são alvo de preocupação mundial quanto ao seu tratamento e destino final. Uma atenção especial tem sido dedicada a um grupo especial de compostos que, apesar de apresentarem efeitos negativos ao ecossistema, não possuem uma legislação ambiental que regulamente seus níveis no meio ambiente, sendo conhecidos como contaminantes emergentes (SILVA, 2011; OLIVEIRA et al., 2011). Entre estes, podemos citar os pesticidas (organoclorados, organofosforados, carbamatos e uréias); fármacos (antibióticos, anti-inflamatório, analgésicos, reguladores lipídicos, etc.); cosméticos e produtos de higiene pessoal (bronzeadores, antissépticos, repelentes de inseto e fragrâncias, etc.); produtos químicos industriais (plastificantes, preservantes, produtos de limpeza, aditivos alimentares, etc.); hormônios naturais e sintéticos, entre outros (NAPOLEÃO, 2011; ERVIM et al, 2009).

Alguns desses compostos são capazes de desregular as funções endócrinas dos seres vivos, sendo conhecidos como desreguladores ou interferentes endócrinos (DEs), ou seja, agentes exógenos capazes de afetar o mecanismo de ação dos hormônios naturais produzidos pelas glândulas endócrinas, bloqueando e/ou mimetizando sua ação e as funções vitais do organismo. Após a síntese, os hormônios são levados aos órgãos pela corrente sanguínea, ligando-se especificamente às células receptoras. Entretanto, quando os DEs interagem com estas células, ocorre uma alteração na resposta esperada, ocasionando o desequilíbrio do sistema imunológico (GHISELLI e JARDIM, 2007; OLIVEIRA et al. 2011).

Entre a grande variedade de compostos com suposta ação disruptora, podemos citar o corante Ponceau 4R (E-124), ilustrado na Figura 1, o qual está inserido no grupo dos azo- 
compostos, ou seja, compostos que possuem o grupo funcional $\mathrm{R}_{1}-\mathrm{N}=\mathrm{N}-\mathrm{R}_{2}$, onde $\mathrm{R}_{1}$ e $\mathrm{R}_{2}$ são grupos alquílicos e/ou arílicos (BAZIN et al., 2013). Trata-se de um corante artificial de intensa coloração avermelhada, considerado um importante aditivo sem valor nutritivo, usado na indústria de alimentos (balas, doces, refrescos, bebidas e enlatados); na coloração de alguns medicamentos; além do tingimento de tecidos (SOUZA, 2012; PRADO e GODOY, 2003). No Brasil, o E-124 tem seu uso regulamentado pela Agência Nacional de Vigilância Sanitária de Alimentos - ANVISA (BENINCÁ, 2012), mas o mesmo tem sido banido de alguns países como Estados Unidos e Finlândia, por consta dos seus efeitos nocivos à saúde.

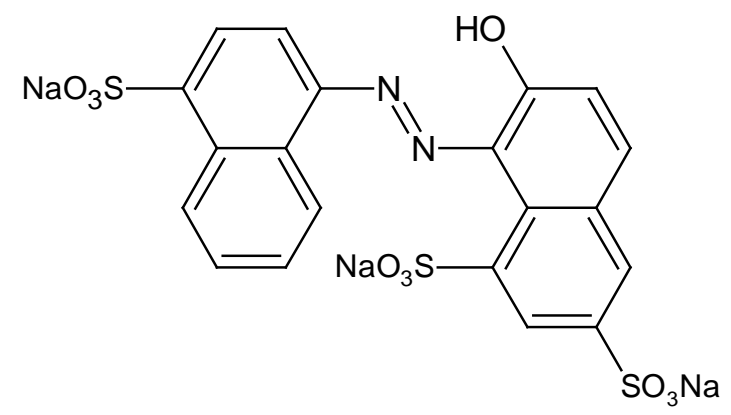

Figura 1 - Estrutura química do azo-corante Ponceau 4R (E-124).

Muitos métodos analíticos têm sido propostos para a análise e controle de qualidade do E-124, tais como cromatografia em camada delgada, cromatografia em coluna acoplada à detector de arranjo de diodos ou espectrômetro de massa, eletroforese capilar, espectrofotometria derivativa, entre outros. Entretanto, os métodos cromatográficos são dispendiosos e instrumentação de complexa manipulação (ZHANG et al., 2014; SAYAR e ÖZDEMIR, 1998). Os métodos espectroscópicos apresentam maior susceptibilidade à interferentes e possuem uma série de restrições para análises de rotina (ZHANG et al., 2014; WANG et al., 2015).

Para contornar estas limitações, muitos pesquisadores têm se dedicado ao desenvolvimento e aplicação de métodos eletroanalíticos de análise. Esses procedimentos são mais robustos e menos susceptíveis a interferentes naturais e antrópicos presentes nas amostras, possibilitando, em alguns casos, experimentos in situ de diferentes contaminantes. Também permitem ensaios com amostras coloridas e/ou com partículas suspensas. A associação entre os resultados obtidos e os critérios de diagnósticos de cada técnica fornece informações que vão além da análise trivial dos poluentes, a exemplo da aquisição de parâmetros cinéticos, mecanísticos e ocorrência de reações paralelas (OLIVEIRA, 2013).

Com o avanço de pesquisas envolvendo materiais condutores, uma grande variedade de sensores vem sendo desenvolvidos, com características e vantagens distintas, que atribuem maior sensibilidade aos procedimentos. Os sensores são instrumentos adequados à detecção de compostos químicos utilizando sinais elétricos, térmicos ou ópticos (MULLER, 2008). Sua modificação por diferentes materiais, a exemplo de compostos inorgânicos derivados de ferro, cobalto, níquel, rutênio, etc., atribui uma nova definição ao eletrodo de trabalho, conhecida como Eletrodos Quimicamente Modificados (EQMs). O termo EQM foi introduzido na eletroquímica por MURRAY et al. (1984) para designar eletrodos com espécies quimicamente ativas, devidamente imobilizadas em suas superfícies, com o objetivo de pré-estabelecer e controlar a natureza físico-química da interface eletrodo/solução. A modificação da superfície do eletrodo atribui características físico-químicas especiais aos dispositivos, a exemplo da reatividade e/ou seletividade, possibilitando o desenvolvimento de sensores com respostas cada vez mais sensíveis e específicas para vários propósitos e aplicações (BERNADELLI, 2009). Segundo GALLI et al. (2006), no processo eletroanalítico, o analito de interesse reage com a superfície eletródica, onde acontece a transferência de elétrons. Se o processo eletródico for muito lento, ele poderá não ser observado, dependendo da velocidade de varrimento, ou poderá ocorrer em um valor de potencial fora do alcançado pelo eletrodo de trabalho. A modificação na 
superfície eletródica pode superar estas limitações, contribuindo positivamente com a cinética de transferência eletrônica.

Entre os estudos eletroanalíticos desenvolvidos para o E-124, podemos citar o reportado por WANG et al. (2015), onde um eletrodo de carbono vítreo modificado com um compósito de polipirrol e nanotubos de carbono foi utilizado para determinar isômeros dos corantes Amaranto e E-124, presentes em sucos industrializados. Estes isômeros também foram alvo dos estudos desenvolvidos por ZHANG et al. (2014), cujas análises destes em sucos de uva foram feitas com um eletrodo compósito contendo grafite expandida. COMBEAU et al. (2002) utilizaram um eletrodo gotejante de mercúrio para determinar simultaneamente E-124, Azorubina e Vermelho Allura em xaropes, refrigerantes e bebidas não alcoólicas. Todos os sensores acima relatados apresentam alta sensibilidade para a detecção do E-104, mas apresentam limitações quanto ao custo, estabilidade, toxicidade e passivação da superfície.

Conhecendo as potencialidades dos EQMs formados por filmes inorgânicos, o presente trabalho tem por objetivo desenvolver um método eletroanalítico para E-124, utilizando um eletrodo de pasta de carbono quimicamente modificado com hexacianoferrato de níquel (NiHCF-EPC), visando sua determinação em águas naturais contaminadas por este corante.

\section{Metodologia}

\subsection{Reagentes e soluções}

Todos os reagentes utilizados neste estudo foram de pureza analítica. Uma solução do E-124 com concentração de $1,0 \times 10^{-3} \mathrm{~mol} \mathrm{~L}^{-1}$ foi preparada em meio aquoso e utilizada como solução estoque. Para os ensaios eletroanalíticos, utilizou-se uma solução-tampão fosfato $(\mathrm{pH} 7,5)$ como eletrólito de suporte. Todas as soluções foram preparadas a temperatura ambiente $\left(25^{\circ} \mathrm{C}\right)$ e utilizando água ultrapura, obtidas de um sistema Purelab Milli-Q (Analítica).

\subsection{Instrumentação}

Os experimentos eletroquímicos foram realizados em um Potenciostato $\mu$-Autolab, modelo Type III (Methrom) e controlado por um computador, usando o programa NOVA versão 1.10, acoplado a uma célula eletroquímica com capacidade para $10 \mathrm{~mL}$, composta por um sistema de três eletrodos: (1) o NiHCF-EPC como eletrodo de trabalho, (2) um eletrodo de platina como eletrodo auxiliar e (3) um eletrodo de $\mathrm{Ag} / \mathrm{AgCl} / \mathrm{Cl}^{-}$saturato como eletrodo de referência.

\subsection{Construção do eletrodo modificado}

Para a construção do eletrodo de trabalho, foi utilizado um eletrodo de pasta de carbono como suporte eletródico para a modificação, composto de pó de grafite ultrapuro e óleo mineral (nujol), na proporção 75:25 (m/m), respectivamente. O material resultante foi embutido na cavidade de um tubo de polietileno, com 5,0 mm de diâmetro, sendo o contato elétrico estabelecido por um fio de aço-inox. Este dispositivo foi imerso em uma solução mista, composta por $\mathrm{K}_{3} \mathrm{Fe}(\mathrm{CN})_{6}$ a $1,0 \times 10^{-3} \mathrm{~mol} \mathrm{~L}^{-1}, \mathrm{NiCl}_{2}$ a $1,0 \times 10^{-3} \mathrm{~mol} \mathrm{~L}^{-1}$ e $\mathrm{KCl}$ a $0,2 \mathrm{~mol} \mathrm{~L}^{-1}$. O filme de hexacianoferrato de níquel foi obtido pelo método galvanostático, por meio de varreduras consecutivas de potencial $\quad\left(0,0-1,0 \mathrm{~V}\right.$; vs. $\mathrm{Ag} / \mathrm{AgCl}^{-} \mathrm{Cl}^{-}$saturato $)$a uma velocidade de $50 \mathrm{mV} \mathrm{s}^{-1}$.

\subsection{Determinação eletroanalítica}

Após conhecer o comportamento eletroquímico do corante E-124 sobre o eletrodo de trabalho com e sem modificação, sua determinação eletroanalítica foi feita pela técnica de cronoamperometria, aplicando-se um potencial elevado o suficiente para que a oxidação do mesmo ocorresse, permitindo o monitoramento dos valores de corrente ao longo do tempo, após sucessivas adições do corante à célula eletroquímica. Este procedimento permitiu a construção de curvas analíticas, a partir da relação entre a corrente observada e a concentração do analito presente na célula. Utilizando a equação de regressão linear obtida, foi possível inferir a concentração do corante E-124 presente em águas naturais artificialmente contaminadas. 


\subsection{Aplicação do procedimento}

O procedimento eletroanalítico proposto foi utilizado para determinar a concentração do corante E-124 em amostras de águas naturais, coletadas em diferentes trechos da Bacia Hidrográfica Apodi/Mossoró (Governador Dix-Sept Rosado, Mossoró e Areia Branca), com o intuito de avaliar a influência da matéria orgânica na aplicabilidade do método. Antes das análises, as amostras passaram apenas por um processo de filtração simples, utilizando papel quantitativo $(4,0 \mu \mathrm{m})$. Em seguida, foi feita uma contaminação artificial das amostras com o corante em três níveis de fortificação $\left(10^{-5}, 10^{-4}\right.$ e $10^{-3} \mathrm{~mol} \mathrm{~L}^{-1}$ de E-124). A eletroanálise dessas amostras permitiu avaliar o nível de acurácia do método.

\section{Resultados e Discussão}

\subsection{Modificação do eletrodo}

Inicialmente, a atenção do trabalho foi direcionada para a construção do eletrodo modificado pelo método galvanostático. O processo de modificação foi assistido por voltametria cíclica, através de varreduras consecutivas de potencial na faixa de 0,0 a $1,0 \mathrm{~V}$ (vs. Ag/AgCl/Cl ${ }^{-}$saturato), obtidas a $50 \mathrm{mV} \mathrm{s}^{-1}$, usando uma solução mista, formada por $\mathrm{K}_{3} \mathrm{Fe}(\mathrm{CN})_{6}$ a $1,0 \quad 3 \mathrm{~mol} \mathrm{~L} \mathrm{~L}^{-1}, \mathrm{NiCl}_{2}$ a 1,0 3 $\mathrm{mol} \mathrm{L}^{-1}$ e $\mathrm{KCl}$ a $0,2 \mathrm{~mol} \mathrm{~L}^{-1}$, como eletrólito. O êxito da formação do filme e consequentemente, da modificação da superfície do eletrodo de pasta de carbono pode ser constatado pelo surgimento de dois pares redox reversíveis e bem definidos, conforme mostrado na Figura 2. Este perfil voltamétrico, formado por dois picos anódicos (picos 1 e 2) e dois picos catódicos (picos 3 e 4), é reflexo de alterações no estado de oxidação dos átomos de ferro presentes no complexo metálico, cujas reações podem ser descritas como:

$$
\begin{aligned}
& \left.\mathrm{K}_{2} \mathrm{Ni}^{\mathrm{II}}\left[\mathrm{Fe}^{\mathrm{II}} \mathrm{CN}\right)_{6}\right] \leftrightarrow \mathrm{KNi}^{\mathrm{II}}\left[\mathrm{Fe}^{\mathrm{III}}(\mathrm{CN})_{6}\right]+\mathrm{e}^{-}+\mathrm{K}^{+} \\
& \mathrm{KNi}^{\mathrm{II}}{ }_{1.5}\left[\mathrm{Fe}^{\mathrm{II}}(\mathrm{CN})_{6}\right] \leftrightarrow \mathrm{Ni}^{\mathrm{II}}{ }_{1.5}\left[\mathrm{Fe}^{\mathrm{III}}(\mathrm{CN})_{6}\right]+\mathrm{e}^{-}+\mathrm{K}^{+}
\end{aligned}
$$

De acordo com KARNJANAKOM et al. (2014), os eventos de oxidação de $\mathrm{Fe}^{2+}$ a $\mathrm{Fe}^{3+}$ observados, paralelos à liberação de $\mathrm{K}^{+}$para o seio da solução, se relacionam com os picos anódicos observados durante a eletrodeposição do filme inorgânico, enquanto que os processos de redução de $\mathrm{Fe}^{3+}$ a $\mathrm{Fe}^{2+}$ ocorre também a absorção de $\mathrm{K}^{+}$a partir da solução, estão relacionados com os picos catódicos, justificando os dois pares redox observados para o hexacianoferrato de níquel.

Como pode ser observado, com o aumento do número de ciclos, há um aumento nas correntes dos picos redox, sugerindo aumento na espessura do filme eletrodepositado sobre a superfície do eletrodo de pasta de carbono, bem como da cinética de transferência eletrônica. Entretanto, constatou-se que após 30 ciclos, os valores de corrente aumentam de forma nãolinear, indicando aumento na resistência de transferência eletrônica $\left(\mathrm{R}_{\mathrm{tc}}\right)$ que, por sua vez, está diretamente relacionada com a espessura do filme formado. $O$ aumento na $R_{t c}$ afeta negativamente a resposta eletroquímica para fins analíticos, por diminuir a intensidade das correntes observadas para os diferentes processos redox que ocorrem na interface eletrodo/solução. Sendo assim, o eletrodo quimicamente modificado empregado nos estudos eletroanalíticos, definido como NiHCF-EPC, foi formado após 30 varreduras consecutivas de potencial, nas condições acima descritas. 


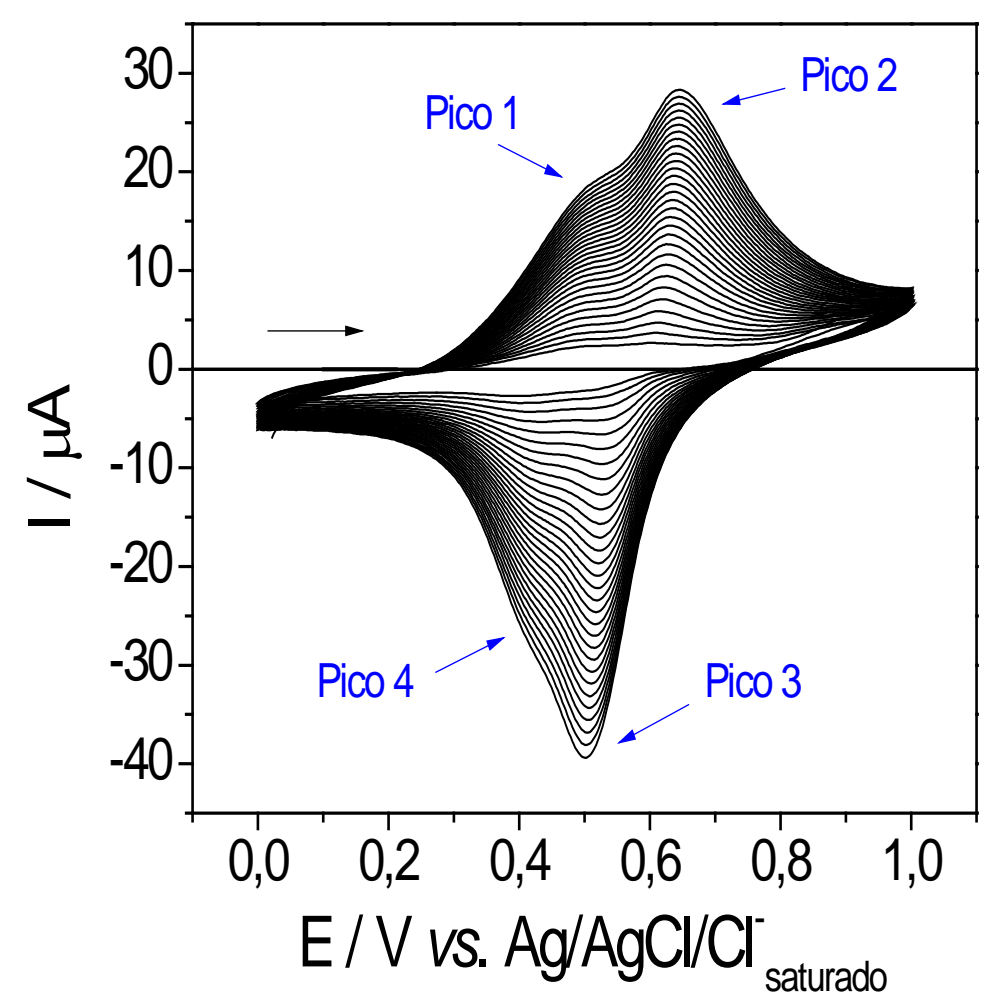

Figura 2 - Voltamogramas cíclicos consecutivos obtidos para a modificação do eletrodo de pasta de carbono, em meio de solução mista de $\mathrm{K}_{3} \mathrm{Fe}(\mathrm{CN})_{6}$ a $1,0 \times 10^{-3} \mathrm{~mol} \mathrm{~L}^{-1}, \mathrm{NiCl}_{2}$ a $1,0 \times 10^{-3} \mathrm{~mol} \mathrm{~L}^{-1}$ e $\mathrm{KCl}_{\text {a }}$ $0,2 \mathrm{~mol} \mathrm{~L}^{-1}$, empregando $50 \mathrm{mV} \mathrm{s}^{-1}$.

\subsection{Estabilidade do NiHCF-EPC}

Variando a velocidade de varredura entre 10 e $500 \mathrm{mV} \mathrm{s}^{-1}$, para uma faixa de potencial entre 0,0 e $1,0 \mathrm{~V}$ (vs. $\mathrm{Ag} / \mathrm{AgCl} / \mathrm{Cl}^{-}$saturato), também ficou constatado um aumento nos valores das correntes de pico $\left(\mathrm{I}_{\mathrm{p}}\right)$ para todos os processos, além dos deslocamentos dos potenciais de pico anódicos para valores mais positivos e no sentido oposto para os picos catódicos. Acima de $200 \mathrm{mV} \mathrm{s}^{-1}$, além do aumento das correntes faradáicas, também se observa grande contribuição de correntes capacitivas e, na medida em que a velocidade de varredura aumenta apenas um par redox passa a ser observado, indicando que a velocidade de varredura pode ter forte influência sobre a formação e estabilidade do filme. De fato, quanto maior a velocidade de varredura empregada para a imobilização do filme, a estabilidade do mesmo era reduzida, bem como a seletividade e intensidade dos processos. Por esta razão, optou-se por trabalhar com uma velocidade de $50 \mathrm{mV}$ $\mathrm{s}^{-1}$ para a modificação do eletrodo de trabalho.

Ainda com relação à estabilidade, esta propriedade foi avaliada por meio de 200 varreduras consecutivas de potencial, empregando voltametria cíclica a $50 \mathrm{mV} \mathrm{s}^{-1}$, usando uma solução de $\mathrm{KCl}$ a $0,2 \mathrm{~mol} \mathrm{~L}^{-1}(\mathrm{pH} \mathrm{6,7)} \mathrm{como} \mathrm{eletrólito} \mathrm{de} \mathrm{suporte.} \mathrm{Os} \mathrm{resultados} \mathrm{mostram} \mathrm{uma}$ alta estabilidade do filme de hexaciaferrato de níquel, uma vez que após o estudo acima descrito, os valores de corrente tiveram uma redução média de apenas 3,4\% do valor inicial.

\subsection{Determinação eletroanalítica do corante E-124}

O sinal eletroquímico observado para o E-124 sobre NiHCF-EPC é representado por um pico anódico de baixa intensidade, registrado em $0,7 \mathrm{~V}$ ( $v s$. $\mathrm{Ag} / \mathrm{AgCl} / \mathrm{Cl}^{-}{ }_{\text {saturato }}$ ). Por meio de varreduras consecutivas de potencial, também foi constatado que este processo tem características adsortivas, em virtude da diminuição da intensidade das correntes com o aumento do número de ciclos. Todavia, levando em consideração a elevada estabilidade do filme e 
considerando que este corante e seus produtos de oxidação são solúveis em meio etanólico, observou-se que a simples lavagem do eletrodo em etanol sob constante agitação, por um período de 5 minutos foi suficiente para remover os produtos polimerizados e reativar a resposta do sensor.

A determinação eletroquímica do E-124 foi feita por cronoamperometria, aplicando um

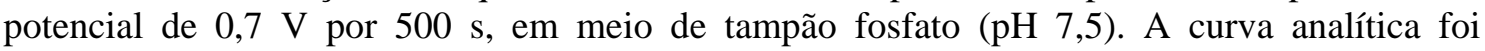
construída pelo decaimento nos valores de corrente, à medida que aumentava a concentração de E-124, conforme apresentado na Figura 3A.

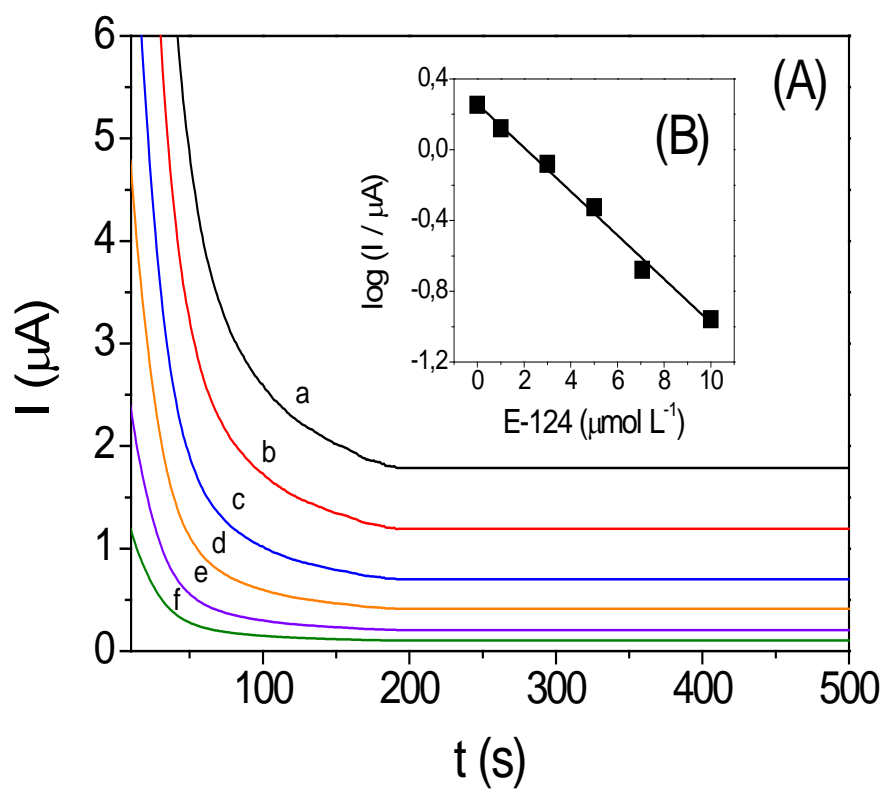

Figura 3 - (A) Curvas cronoamperométricas para a eletrooxidação de E-124 sobre NiHCF-EPC, nas concentrações de (a) 1 , (b) 3, (c) 5, (d) $7 \mathrm{e} \mathrm{(e)} 10 \mu \mathrm{mol} \mathrm{L}^{-1}$, aplicando um potencial de 0,7 V por $500 \mathrm{~s}$, em meio de tampão fosfato (pH 7,5). (B) Curva analítica obtida a partir dos cronoamperogramas.

Foi observada uma relação linear entre o logaritmo das correntes em função da concentração do corante, para uma faixa de $1,02 \times 10^{-6}$ a $1,23 \times 10^{-5} \mathrm{~mol} \mathrm{~L}^{-1}$, com limite de detecção de $9,90 \times 10^{-7} \mathrm{~mol} \mathrm{~L}^{-1}$ e um coeficiente descrito como:

$$
\log I(\mu \mathrm{A})=0,25-1,24 \times 10^{5} \times[\mathrm{E}-124]\left(\mathrm{mol} \mathrm{L}^{-1}\right)
$$

A precisão das medidas foi constatada por testes de repetibilidade, para uma concentração de $7,02 \times 10^{-6} \mathrm{~mol} \mathrm{~L}^{-1}$ do corante, resultando em um desvio padrão relativo de 7,41\%. Quanto à reprodutibilidade, analisada pelas medidas de três diferentes dispositivos construídos sob as mesmas condições otimizadas, o desvio padrão relativo foi equivalente à 8,23\%. A passivação da superfície do sensor pode ser um dos principais agravantes para a diminuição na precisão das medidas. De um modo geral, os dados analíticos obtidos mostram a viabilidade deste sensor para a determinação de E-124 em matrizes diversas.

\subsection{Aplicação em amostras de águas naturais}

Após otimização do protocolo analítico, o procedimento eletroanalítico proposto foi aplicado para identificar e determinar o corante em amostras de águas naturais artificialmente contaminadas $\left(7,02 \times 10^{-6} \mathrm{~mol} \mathrm{~L}^{-1}\right)$, através do método da adição de padrão. Conforme pode ser observado na Tabela 1, os níveis de recuperação variaram de 85,9 a $104.2 \%$, sendo que a diminuição na exatidão diminui com o aumento dos níveis de matéria orgânica presente na amostra. Isso ocorre porque a matéria orgânica também é passível de oxidação sobre NiHCF- 
EPC, passando a concorrer com o E-124 pelos sítios ativos do eletrodo. Em todo caso, os resultados obtidos dão credibilidade para a utilização de NiHCF-EPC como dispositivo analítico, embora estudos mais detalhados ainda sejam necessários a fim de atribuir maior exatidão ao método eletroanalítico proposto.

Tabela 1 - Informações relacionadas aos ensaios de recuperação de E-124 em amostras de águas naturais, utilizando NiHCF-EPC como sensor de trabalho.

\begin{tabular}{cccc}
\hline Ponto de Coleta & $\begin{array}{c}\text { Carbono orgânico } \\
\text { Total (mg L }\end{array}$ & $\begin{array}{c}\text { Fortificação com E- } \\
\mathbf{1 2 4}\left(\mathbf{m o l ~ L}^{-\mathbf{1}}\right)\end{array}$ & Recuperação (\%) \\
\hline Governador Dix-Sept & 9,3 & & 104,2 \\
Rosado & 18,4 & $7,02 \times 10^{-6}$ & 97,2 \\
Mossoró & 27,8 & & 85,9 \\
Areia Branca & & & \\
\hline
\end{tabular}

\section{Conclusões}

O método eletroanalítico proposto, empregando NiHCF-EPC como sensor de trabalho, mostrouse eficiente para a detecção e quantificação de E-124 em amostras de águas naturais. O eletrodo modificado apresentou alta estabilidade e sensibilidade para a detecção do respectivo corante. Os testes de precisão e exatidão das medidas reforçam a aplicabilidade do dispositivo para fins analíticos, embora estudos mais detalhados ainda sejam necessários, a fim de melhorar o desempenho deste sensor para analisar o corante em amostras com elevado teor de matéria orgânica.

\section{Electroanalysis of Ponceau 4R using a nickel hexacyanoferrate modified carbon paste electrode}

\section{Abstract:}

The worsening of environmental problems from the industrial activities increased the demand for new monitoring technologies for the generated pollutants. There is a special attention with the industries that use dyes as raw material, since these compounds can disrupt seriously the ecosystems and affect the health of living beings, when disposed improperly. The azocompounds, such as Ponceau 4R (E-124), are among the most widely employed in textile, pharmaceutical and food sectors, showing the relevance of new methods for the detection and quantification of these potential contaminants. In this work, a nickel hexacyanoferrate carbon paste modified electrode (Ni-HCF/EPC) was used for the E-124 electroanalysis in natural waters. Among the main advantages seen for this device include the high stability, sensitivity and accuracy for the analysis of the dye. Using chronoamperometry as electroanalytical technique, it was possible to quantify E-124 with a detection limit of $9.90 \times 10^{-7} \mathrm{~mol} \mathrm{~L}^{-1}$. The percent recovery obtained in spiked natural waters, containing different levels of organic matter, ranged from 85.9 to $104.2 \%$, showing the applicability of the proposed procedure.

Keywords: Natural waters; Azo-compounds; Ponceau 4R; Chemically modified electrodes; Electroanalysis. 
Referências Bibliográficas

Agencia Nacional de vigilância sanitária. disponível em URL: http://www.anvisa.gov.br/anvisalegis/resol/44_77.htm Acesso em 03/06/2015 as 01:01h BAZIN, Ingrid, HASSINE, Aziza ibn Hadj, GONZALES, Wissem Mnif and Catherine.YES as a Tool for Detecting Estrogenic Activity of Some Food Additives Compounds: E 104, E 122, E 124, E 132 and E 171. J. Ecosys. Ecograph., 2013, vol 3, n 3.

BENINCÁ, Cristina. Degradação do corante alimentício ponceau 4R e tratamento de efluente de uma indústria de alimentos utilizando processos oxidativos avançados, Universidade Federal do Paraná. Curitiba: 2012.

BERNADELLI, JOSSI KARLA BRASIL. Preparo e caracterização de eletrodo a base de carbono bismuto e poliuretano para determinação de metais, Universidade Tecnológica Federal do Paraná. Curitiba: 2009.

COMBEAU,S, CHATELUT,M, VITTORI O.Identification and simultaneous determination of Azorubin, Allura red and Ponceau 4R by differential pulse polarography: application to soft drinks. Talanta, Volume 56, Issue 1, 4 January 2002, Pages 115-122.

GIRARD, James E.Tradução Marcos José de Oliveira; revisão técnica Tadeu Grass. Princípios de Química ambiental. Rio de Janeiro, 2013.

GUARATINI, Cláudia C. I. ZANONI, Maria Valnice B. Corantes têxteis. Departamento de Química Analítica - Instituto de Química - UNESP - 14800-900 Araraquara - SP.2000.

GHISELLI, Gislane, JARDIM, Wilson F. Interferentes endócrinos no meio ambiente. Química Nova, No.3. v. 30, p. 695-706, 2007.

KARNJANAKOM, Surachai, MA, Yufei, GUAN, Guoqing, PHANTHONG, Patchiya, HAO, Xiaogang, DU, Xiao, SAMART ,Chanatip, ABUDULA, Abuliti. Fabrication of nickel hexacyanoferrate film on carbon fibers by unipolar pulse electrodeposition method for electrochemically switched ion exchange application. Electrochimica Acta 139 (2014) 36-41 NAPOLEÂO, Daniela Carla. Avaliação e tratamento dos contaminantes emergentes (ácido acetilsalicílico, diclofenaco, paracetamol) usando processos oxidativos. Dissertação de mestrado em Engenharia Química, Universidade Federal de Pernambuco, Recife 2011.

OLIVEIRA, Thiago M. B. F. Biossensores enzimáticos para detecção e quantificação de carbonato em amostras de alimentos. Tese de Doutorado programa de pós-graduação em química, Universidade Federal de Ceará, Fortaleza 2013.

PRADO, Marcelo Alexadre, Godoy, Helena Teixera. Corantes artificiais em alimentos. Alimentos e nutrição, Araraquara, v.14, n.2, p. 237-250, 2003.

SAYAR, Sedat, ÖZDEMIR, Yüksel. First-derivative spectrophotometric determination of ponceau $4 \mathrm{R}$, sunset yellow and tartrazine in confectionery products. Food Chemistry, $\underline{61}$ (1998) 367-372.

SILVA, Rogério. Avaliação da presença de contaminantes emergentes em tratamento de esgoto do Estado de Pernambuco e suas degradações por POA. Dissertação de mestrado do curso de Química, Universidade Federal de Pernambuco, Recife: 2011.

SOUZA, Rosilane Moreth. Corantes naturais e seus benefícios à saúde. Trabalho de conclusão de curso de Farmácia, Centro Universitário Estadual da Zona oeste, Rio de Janeiro: 2012.

WANG, Zifei, ZHANG ,Hui, WANG, Zhipeng, ZHANG ,Jie, DUAN, Xuemin, XU, Jingkun, WEN, Yangping.Trace analysis of Ponceau $4 R$ in soft drinks using differential pulse stripping voltammetry at SWCNTs composite electrodes based on PEDOT:PSS derivatives. Food Chemistry, Volume 180, 1 August 2015, Pages 186-193.

WANG, Meiling, GAO, Yunqiao, SUN, Qian, ZHAO, Jianwei. Ultrasensitive and simultaneous determination of the isomers of Amaranth and Ponceau 4R in foods based 
on new carbon nanotube/polypyrrole composites.Food Chemistry, Volume 172, 1 April 2015, Pages 873-879

ZHANG,Jing, WANG, Meiling, SHENTU, Chao WANG, , Wenchang. CHEN, Zhidong. Simultaneous determination of the isomers of Ponceau 4R and Amaranth using an expanded graphite paste electrode Food Chemistry, Volume 160, 1 October 2014, Pages 1115 